\begin{tabular}{|c|ccc|c|}
\hline & PORT SAID ENGINEERING RESEARCH JOURNAL \\
\hline
\end{tabular}

\title{
Investigation of the Flow Behavior of Solid-Liquid Settling Slurries in a Horizontal Pipeline
}

\begin{abstract}
Tamer N. Mahmoud ${ }^{1}$, Imam A. El-Sawaf ${ }^{2}$ and Kamal E. El-Nahhas ${ }^{3}$

Developing accurate models for pressure drop in slurry pipelines is one of the most important challenges of researchers around the world. Pressure drop is one of the most important technical parameters to be evaluated by the designer of pipeline slurry transportation system. Also it is the parameter which dictates the selection of pump capacity. The properties of slurries mainly depend on the tendency of the particles to settle out from the carrying liquid. The extent to which the particles will be free to settle in horizontal flow depends on the terminal velocity, the turbulence and particle particle interaction. Physical models for the prediction of the pressure drop, flow regime, and specific energy consumption are presented. Several studies for pressure drop prediction in slurry flow are available in literature. The comparis on between the proposed correlations with the experimental data of the settling slurries is satisfactory. Since the decrease of the specific power consumption and increase overall the transport system efficiency, the carrying capacity should be increased. The experimental investigations were performed on three different sorts of sand solids to satisfy the economic advantage by adjusting the particle size. The experiments were conducted in $26.8 \mathrm{~mm}$ inner diameter horizontal pipe on three sizes of sand particles of which mean diameter are $0.2 \mathrm{~mm}$ (fine), $0.7 \mathrm{~mm}$ (medium) and $1.4 \mathrm{~mm}$ (coarse). Flow velocity was up to $7 \mathrm{~m} / \mathrm{s}$ and overall concentration up to $33 \%$ by volume for each velocity. The effect of slurry velocity and particle concentration on the slurry flow behaviour and pressure drop in the turbulent regime was evaluated.
\end{abstract}

Keywords: Settling slurry, pressure drop, flow regime, specific energy consumption and particle size.

\section{INTRODUCTION}

The present paper discourses the investigation of the behaviour of the hydraulic transport of settling solidliquid slurry with different grain sizes, velocities and concentrations in horizontal pipelines. It summarizes the results of preceding investigations that carried out to describe the flow of sand-water mixtures in pipelines. According to the comparisons between these models with the experimental results it proposes a method of mixture flow prediction. An attention has been paid to methods of pressure loss evaluation and specific energy consumption estimation, as power consumption represents substantial part of pipeline hydraulic transport operational cost.

In slurry practice, the models used to predict the behavior of mixture flow in pipelines are based on an empirical approach rather than on a physical description of the flow mechanis ms. Although the physical approach was introduced in the form of a two-layer model. It has rarely been used in practice. This is because that the model was considered applicable only to the special flow conditions.

Results of the experimental tests show a satisfactory agreement with certain correlations and failed to be explained by other correlations according to the particle size, solid concentration and velocity range. The investigation proved that these correlations are prone to great uncertainty as one departs from the limited database that supports them. Flow behavior of settling slurries has

\footnotetext{
${ }^{1}$ Mechanical Power Department, Faculty of Engineering, Suez Canal University, Ismailia,Egypt, E-mail: tamer_mtc@yahoo.com

${ }^{2}$ Mechanical Power Department, Faculty of Engineering, Port Said

University, Port Said, Egypt,E-mail: iaelsawf@hotmail.com

${ }^{3}$ Suez.Canal Authority, Egypt, E-mail: k_elnahhas@yahoo.com
}

been experimentally studied for three different sorts of sand mono-disperse quartz $(0.2,0.7$ and $1.4 \mathrm{~mm})$ mixed with water at different concentration (from $4 \%$ to $33 \%$ ) in a laboratory pipeline test loop ( $\mathrm{D}=26.8 \mathrm{~mm})$ flowing at different velocity (from $1 \mathrm{~m} / \mathrm{s}$ to $7 \mathrm{~m} / \mathrm{s}$ ).

The most popular empirical models, related to the pressure drop, used nowadays referred to 1950's. Durand (1952) presented a correlation of dimensionless groups contains the most important flow parameters and satisfies excellent agreement with all fine slurries concentrations and some medium slurries according to solid concentration and velocity and didn't match with coarse slurries. From point of view that the flow takes the form of different regimes, from a simple stationary bed at low speeds to a pseudo-homogeneous flow at high speeds Newitt (1955) introduced his correlation but the restricted condition of this correlation makes it limited to use.

Doubts were raised when Zandi and Govatos (1967) showed a large discrepancy of Durand's correlation. Durand correlation seemed to be invalid for the solids transport by saltation, therefore Zandi and Govatos suggested a criterion for separation of the heterogeneous and saltation regimes in a slurry pipeline with individual correlations.

Four distinct regimes were found existent, namely flow with stationary bed, flow with moving bed, heterogeneous suspension and homogeneous suspension. For each regime, equations have been developed over the years. Turian and Yuan (1977) proposed a method for identifying the flow regime and displayed a correlation for each regime. Thus regime identification becomes important for slurry pipeline design as they are the prerequisite to select suitable pressure drop correlation in that regime. 


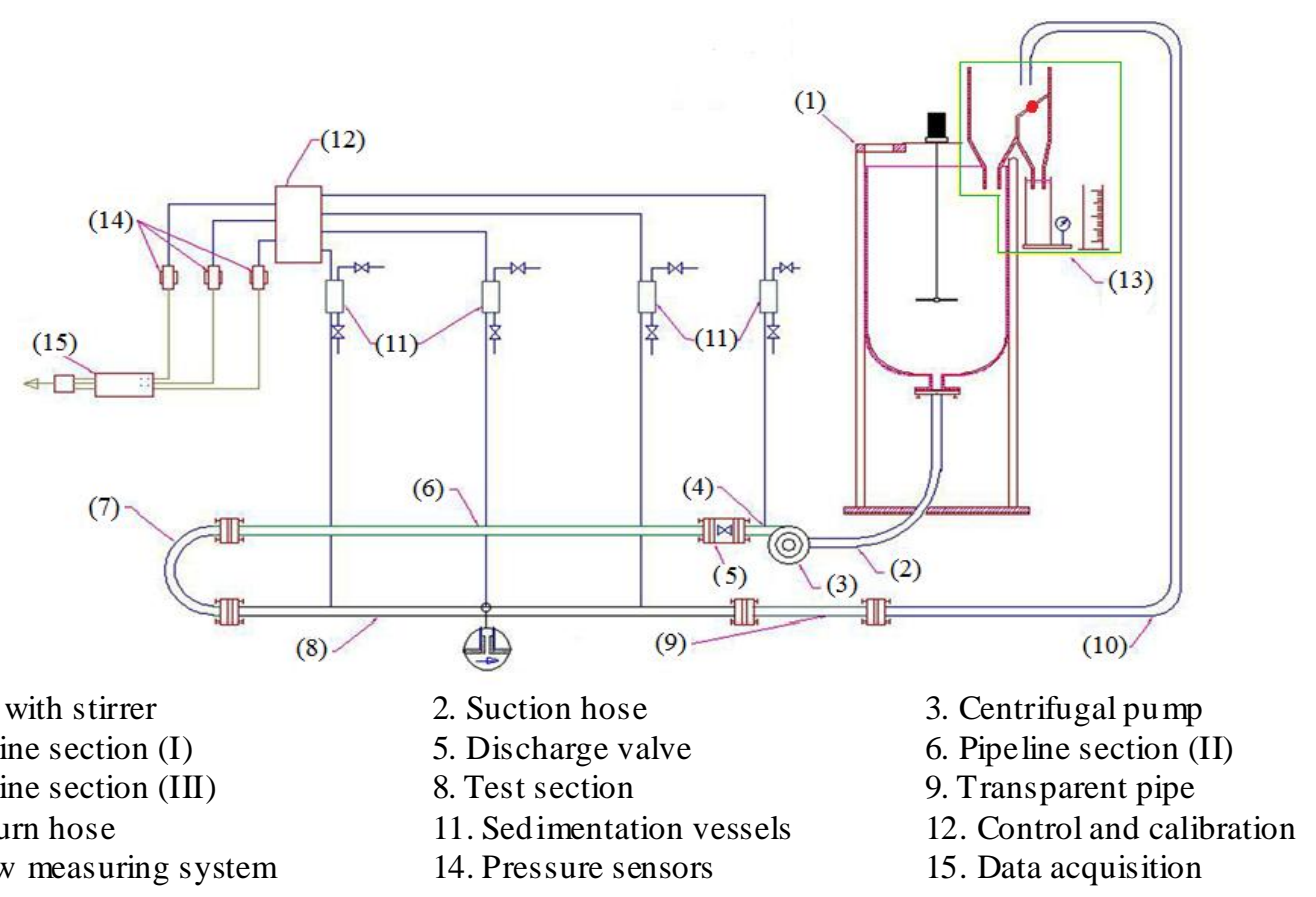

Figure (1) Schematic diagram of the experimental pi peline test loop

Lazarus and Nielsen (1978) suggested a new correlation depend on dimensionless analysis and the mass flow rate ratio. Wilson and Addie (1995) investigated the friction gradient of the settling slurries as stratified regimes. Wilson correlation satisfies excellent agreement with coarse slurries at different concentrations and some medium slurries according to solid concentration and velocity and didn't agree with fine slurries.

In 1970's a theoretical concept was introduced based on the principle of force balances in the two layer pattern of a mixture flow stratified into a bed (contact load) and (suspended load). The two layer model (1996) appeared to be a valuable tool for the prediction of fully-stratified flow but problems arose with the application of the model to a partially-stratified flow. This model is not suitable for the present studied systems especially the fine slurries. Wilson, Addie, Selgren, and Clift (WASC) and simplified WASC (1997) deduced an empirical correlation for head losses of heterogeneous settling slurries. From all previous studied models these two models result in highly over prediction results and maximum solid effect of all studied systems.

\section{EXPERIMENTAL SETUP}

An open-loop recirculation pipeline system, shown schematically in Figure (1), was employed for testing the slurry flow behavior.

A stainless steel pipe loop of internal diameter $26.8 \mathrm{~mm}$ was used for slurry parameters measurement. The test section was located in the back (downstream) branch of the piping loop system. A transparent section was mounted at the end of the test section. Pressure measurements were obtained over two sections of pipe. The pressure is transmitted from the tapping points to three pressure sensors through transmission lines and transparent Perspex sedimentation vessels filled with pure water. The control and calibration unit is used to calibrate the sensitive pressure sensors, control different passes to let the sensors read the pressure of any test point and to protect the pump. Pressure sensors were used to measure the pressure losses between the pressure tappings. The sensors output signals, which is proportional to the applied pressure were displayed as an analogue value (in milli-ampere). Also these analogue signals were converted to digital signals by data acquisition system. The digital data signal is entered to a computer, which is accessed with the LABVIEW software that enables online measurement, analysis and storage the data.

At the downstream end of the test pipes, a box divider was mounted to allow the discharge to be diverted to a plastic container. Since the divider arm was connected to an electrical stopwatch, the slurry mass flow rate, slurry velocity, slurry density and hence the volumetric concentration could be determined, [12]. Three sorts of the mono-disperse quartz sands, $\rho_{\mathrm{s}}=2650 \mathrm{~kg} / \mathrm{m}^{3}$, were used for preparing slurries of the experiments; fine $\left(\mathrm{d}_{50}=0.2 \mathrm{~mm}\right)$, medium $\left(\mathrm{d}_{50}=0.7 \mathrm{~mm}\right)$ and coarse $\left(\mathrm{d}_{50}=1.4\right.$ $\mathrm{mm})$. The solids volumetric concentrations from $\mathrm{C}_{\mathrm{v}}=4 \%$ to $33 \%$. The experimental work was performed through wide range of velocities and measuring the corresponding pressure losses through two pipe sections by three pressure sensors.

\section{MODELING}

Since the first slurry pipelines were built, scientists have tried to correlate the losses with the slurry to those of an equivalent volume of the water. The loss of head per unit pipe length due to friction, called the hydraulic friction gradient. The hydraulic friction gradient of the slurry, $i_{m}$ [m-water/m-pipe], is higher than for an equivalent volume of water. There are number of models to predict the friction losses. Some of them use the drag coefficient while others use the terminal velocity, and the others consider the solids to be moving as a bed and suspended fines above it. The theoretical results uses Churchill equation (1977) for determining Darcy friction factor and hence the hydraulic gradient for water. 
$f=8\left[\left(\frac{8}{R e_{N}}\right)^{12}+\left(A_{c}+B_{c}\right)^{-1.5}\right]^{1 / 12}$

$\mathrm{A}_{c}=\left[-2.457 \ln \left[\left(\frac{7}{\mathrm{Re}_{\mathrm{N}}}\right)^{0.9}+0.27 \frac{\varepsilon}{\mathrm{D}}\right]\right]^{16}$

$\mathrm{B}_{\mathrm{c}}=\left(\frac{37530}{\mathrm{Re}_{\mathrm{N}}}\right)^{16}$

$\operatorname{Re}_{\mathrm{N}}=\frac{\rho_{\mathrm{f}} \mathrm{vD}}{\mu_{\mathrm{f}}}$

$\mathrm{i}_{\mathrm{f}}=\frac{\mathrm{h}_{\mathrm{f}}}{\mathrm{l}}=\frac{\mathrm{fv}^{2}}{2 \mathrm{gD}}$

\subsection{Durand-Condolios Correlation}

Durand equations gained considerable popularity as a design tool since 1952 , but its validity began to be questioned as the corpus of experimental data grew. Durand correlation was constructed using technique of dimensional analysis.

$\Phi=\frac{i_{m}-i_{f}}{C_{v d} i_{f}}$

$\Psi=\frac{V_{m}^{2}}{g D} \frac{\sqrt{g d}}{V_{t}}=\frac{V_{m}^{2}}{g D\left(S_{s}-1\right)} \sqrt{C_{D}}$

A general empirical relationship was established for a resistance due to friction as;

$\Phi=\mathrm{k} \Psi^{n}$

The $\Phi-\Psi$ relationship might be determined using the hyperbolic curve in the $\Phi-\Psi$ plot proposed by Durand or using the curve approximation giving $\mathrm{k}=81, \mathrm{n}=-1.5$. The ultimate correlation obtained by Durand is;

$\frac{\mathrm{i}_{\mathrm{m}}-\mathrm{i}_{\mathrm{f}}}{\mathrm{C}_{\mathrm{vd}} \mathrm{i}_{\mathrm{f}}}=81\left(\frac{\mathrm{V}_{\mathrm{m}}^{2}}{\mathrm{gD}} \frac{\sqrt{\mathrm{gd}}}{\mathrm{V}_{\mathrm{t}}}\right)^{-1.5}$

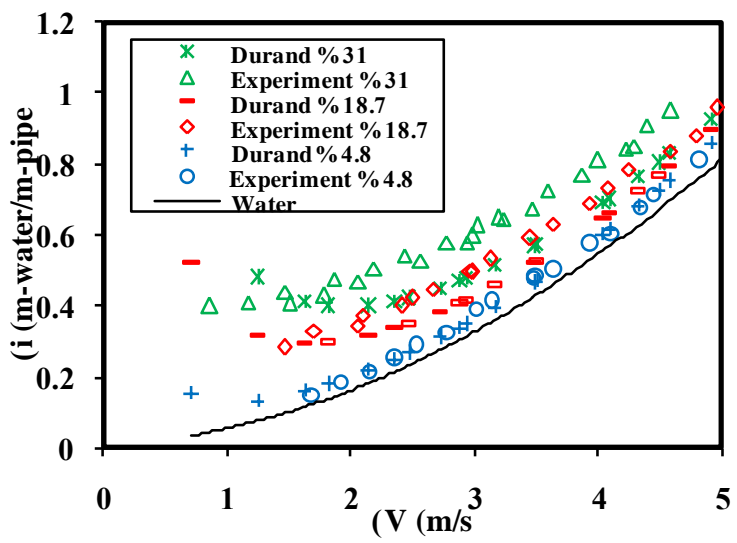

Figure (2) Durand and experimental results of coarse sand slurry at different concentr ations

Durand's formulation gives independence for large heavy particles for which $C_{D}$ is virtually constant so a discrepancy between Durand's prediction and experimental data has been experienced specifically for coarse slurry flow.

In order to compare the merits of different transport systems, it is necessary to have a measure of the energy required to move a given quantity of product over a given distance. This measure is the specific energy consumption
(SEC). The specific energy consumption is therefore to be related to the solids transported rather than to the mixture. The lower the SEC, the more energy-effective the pipeline is as a means of transport.

$\mathrm{SEC}=2.73 \frac{\mathrm{i}_{\mathrm{m}}}{\mathrm{S}_{\mathrm{s}} \mathrm{C}_{\mathrm{vd}}} \quad$ [kw.hr/tonne.km]

Figures (2)-(4) present the experimentally and Durand numerically effects of solid concentration on the resistance curves of different particle size slurries. The general trend is that increasing the mean slurry velocity at certain concentration leads to increasing of the friction losses. Experimental solid effect $\left(i_{m}-i_{f}\right)$ for all different particle slurries is higher than Durand solid effect especially at high concentrations. The solid effect for different slurries is very high at low velocity (stratified regime) and decreases as the slurry mean velocity increases. As the solid concentration decreases the limiting deposition velocity decreases and the experimental and numerical results become closer to each other and approximate to the water behavior so the solid effect consequently decreases. As the solid concentration increase the hydraulic gradient increase at certain slurry velocity. For medium sand slurry, there is a difference between the experimental and numerical results till certain velocity the two behaviours coincide, this coinciding velocity decreases as the concentration decreases.

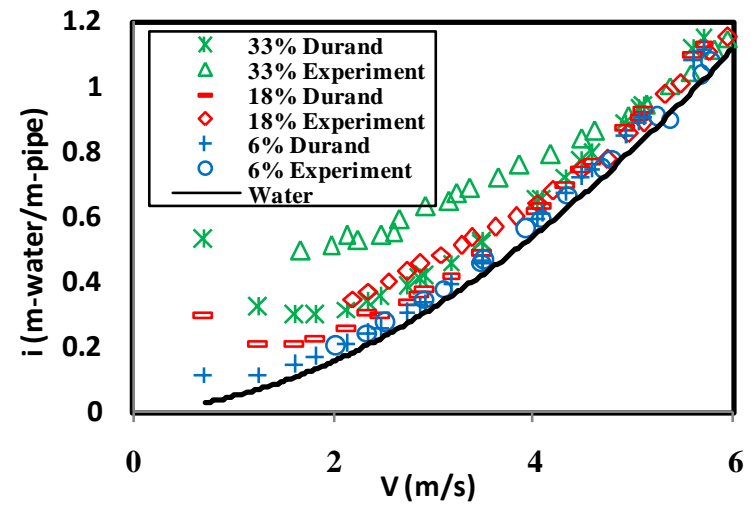

Figure (3) Dur and and experimental results of medium sand slurry at different concentrations



Figure (4) Durand and experimental results of fine sand slurry at different concentrations

The specific energy consumption (SEC) is plotted against the solids throughput, the amount of dry solids delivered at the pipeline outlet over a time period. Figures (5)-(7) show the effect of solid concentration on the 
experimental and numerical relations for coarse, medium and fine sand slurries respectively. At any solid concentration the specific energy consumption increased as the solids throughput increased. As the solids concentration increase the specific energy consumption, for the same solids throughput, decrease. There is an agreement between the experimental and Durand specific energy consumptions at the same solid concentration. Decreasing of specific energy consumption means the increasing of the pipeline hydro-transport efficiency of the sand slurries. The low concentrated sand slurries

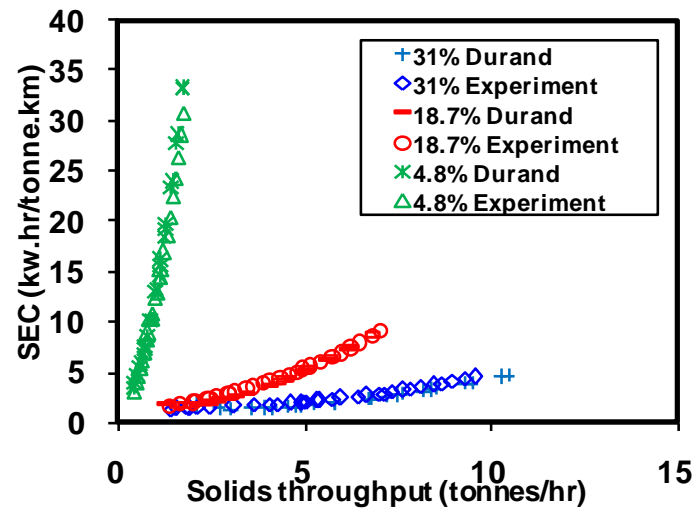

Figure (5) Dur and and experimental specific energy consumptions of coarse slurry at different concentrations



Figure (6) Dur and and experimental specific energy consumptions of medium slurry at different



Figure (7) Durand and experimental specific energy consumptions of fine slurry at different concentrations

hydro-transport in pipelines is available with very limited solids throughputs accompanying with considerably high specific energy.
Figures (8) and (9) compare the Durand resistance curves of fine, medium and coarse-sand slurries at nearly the same solid concentrations. From figures it's obviously shown that the coarse sand slurry has the highest resistance curve. At low slurry velocity the difference between the resistance curves of different sand particle diameter is very large especially at high solid concentration $\left(\mathrm{C}_{\mathrm{V}}=30-33 \%\right)$. At high slurry velocity range the resistance curves are coincide. The overlap between the curves of different particle size at the same concentration starts at velocity value that increases by increasing of solid concentration. It could be noticed that the solid particle size effect on the Durand flow behavior is more significant for slurries with high solid concentration.



Figure (8) Durand resistance curves of different particle sizes slurries



Figure (9) Dur and resistance curves of different particle sizes slurries

\subsection{Zandi and Govatos Correlation}

Doubts were raised when Zandi and Govatos (1967) showed a large discrepancy between Durand's prediction and experimental data for a coarse slurry flow exhibiting considerable stratification. Durand correlation seemed to be invalid for the solids transport by saltation; therefore Zandi suggested a criterion for separation of the heterogeneous and saltation regimes.

$\Phi=\mathrm{k} \Psi^{\mathrm{n}}$

Where;

$$
\begin{array}{lc}
\mathrm{K}=280, \mathrm{n}=-1.93 \text { if } \Psi \leq 10 & \text { (saltation) } \\
\mathrm{K}=6.3, \mathrm{n}=-0.354 \text { if } \Psi>10 & \text { (heterogeneous) }
\end{array}
$$

Zandi and Govatos defined an index nu mber $\mathrm{N}_{\mathrm{e}}$ as;

$$
\mathrm{N}_{\mathrm{e}}=\frac{\mathrm{V}^{2} \sqrt{\mathrm{C}_{\mathrm{D}}}}{\mathrm{gDC}_{\mathrm{vd}}\left(\mathrm{S}_{\mathrm{s}}-1\right)}
$$


When $\mathrm{N}_{\mathrm{e}}<40$ saltation regime occurs but when $\mathrm{N}_{\mathrm{e}} \geq 40$ heterogeneous flow regime develops.

For heterogeneous regime, the index number $\mathrm{N}_{\mathrm{e}} \geq 40$ and also $\Psi>10$ will be illustrated in the following figures of the resistance curves by red color (Zandi\&Govatos II). Every resistance curve consists of saltation part and heterogeneous part connected at deposition velocity point. The saltation part of the curve will be shown by green color (Zandi\& Govatos I).

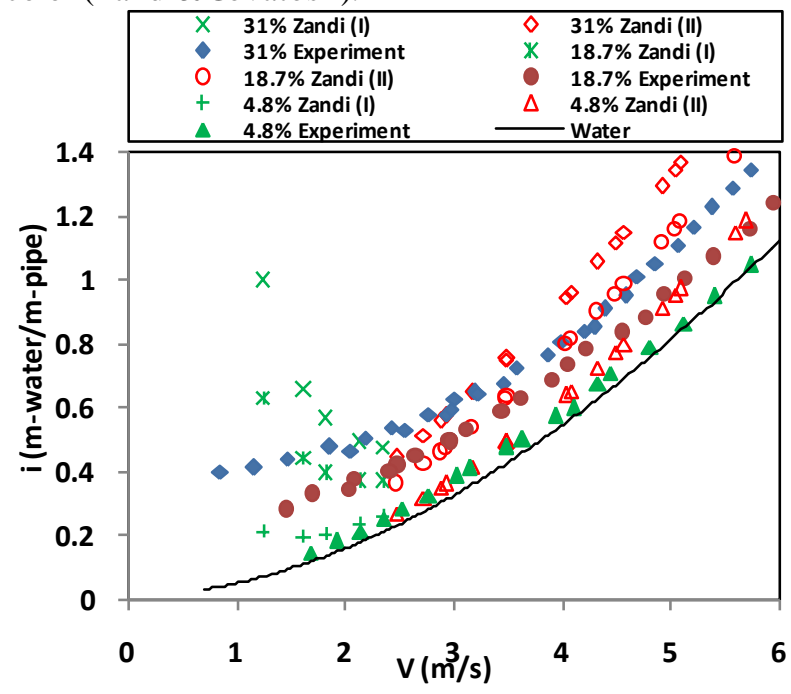

Figure (10) Zandi and experimental results of coarse sand slurry at different concentr ations



Figure (11) Zandi and experimental results of medium sand slurry at different concentrations

Figures (10)-(12) present the effect of solid concentration variation on experimental and Zandi resistance curves. Increasing the slurry velocity leads to the increasing of the pressure losses at certain concentration. The figures show that, at the same slurry mean velocity the hydraulic gradient increase as the solid concentration of the slurry increases. Investigating the figure shows hydraulic gradient of Zandi approaches to the water gradient as the solid concentration decreases. The figure shows Zandi behaviour that for the same solid concentration the hydraulic gradient firstly decreases (stratified regime) and after limit deposition velocity (heterogeneous regime) increases as the slurry velocity increases but the experimental solid effect decreases as the slurry velocity increases. The slope of Zandi behaviour is higher than the experimental one so the computational solid effect is higher than the experimental. Experimental and numerical behaviours come to be close to each other as the concentration decreases. The range of velocity at which the two behaviours overlapped increases as the solid concentration decreases.

For fine particle slurry the range of saltation behavior is extremely narrow to be neglected due to the fine particle of sand tends to be heterogeneous over a wide range of behavior. From all figures and at all concentrations for different sand particle slurries, there are no matching between experimental and Zandi numerical behaviours so this correlation can't be used to explain the behaviour of the studied system.

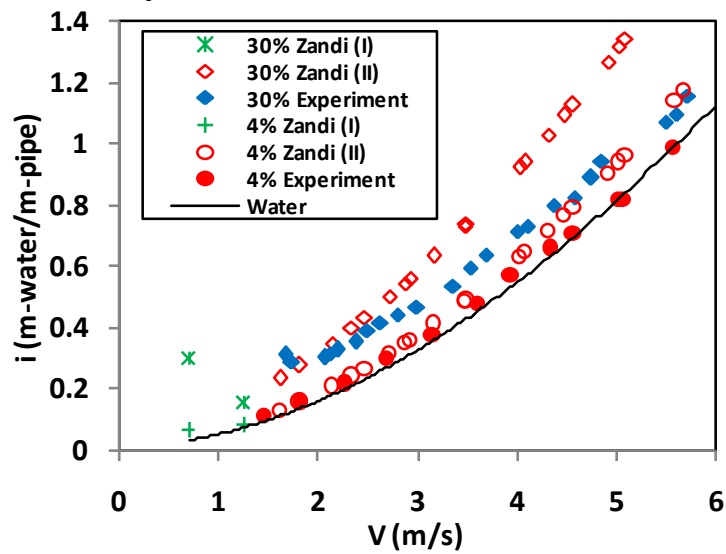

Figure (12) Zandi and experimental results of fine sand slurry at different concentrations

\subsection{Wilson and Addie Correlation}

Wilson and Addie investigated the friction gradient of the settling slurries as fully stratified regimes. Fullystratified flow occurs for coarse particles where almost all of the particles travel as contact load. The ratio of particle diameter to pipe diameter is of major importance in determining the presence of this flow type, which does not normally occur for d/D ratios less than 0.02. Fullystratified flow is less likely if the particles are broadly graded. Fully stratified flow is more likely for larger values of $S_{s}$ and smaller values of $V_{m}$. This type of flow is less energy-efficient than heterogeneous flow.

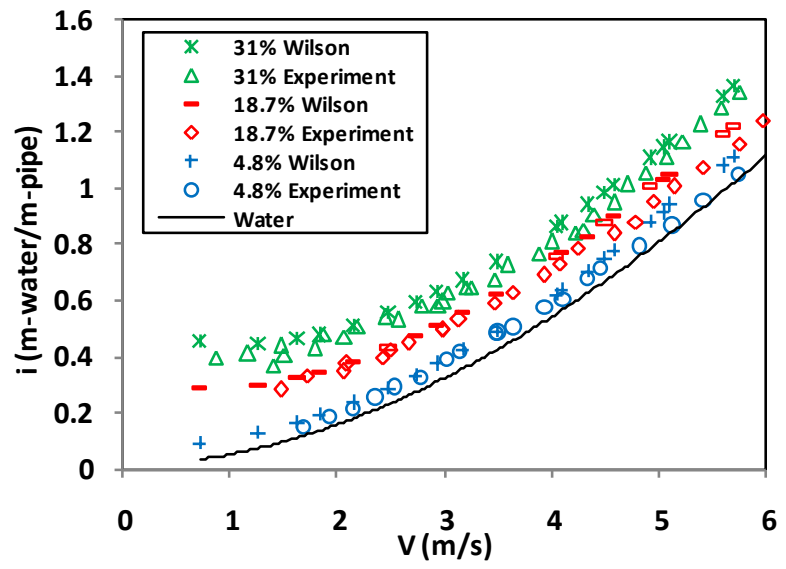

Figure (13) Wilson and experimental results of coarse sand slurry at different concentrations

In dealing with fully-stratified flows it is often desirable to employ a simple method, even if some loss of accuracy may be entailed. The simplest method is that of Newitt, 
this method considers the ratio $\frac{i_{m}-i_{f}}{s_{m}-1}$ to be a constant. In fact, this term is not a constant. Wilson found this relation can be approximated to reasonable accuracy by the expression;

$\frac{\mathrm{i}_{\mathrm{m}}-\mathrm{i}_{\mathrm{f}}}{\mathrm{s}_{\mathrm{m}}-1}=\left(\frac{\mathrm{V}_{\mathrm{m}}}{0.55 \mathrm{~V}_{\mathrm{sm}}}\right)^{-0.25}$

The conservative designer may be content to know only the maximum velocity at the limit of deposition, $V_{\mathrm{sm}}$, since maintaining the operating velocity above this value ensures that deposition will not occur.

For large pipes, and particles near the Murphian size (particles at which the velocity equal the maximum deposition velocity), the new analysis shows that the particle size no longer influences $\mathrm{V}_{\mathrm{sm}}$ directly. Wilson (1992) proposed that the $V_{\text {sm }}$ for fully-stratified flow with the shear layer (marked $\mathrm{V}_{\mathrm{sm} \text {, max }}$ ) should be determined by a power-law approximation, [8];

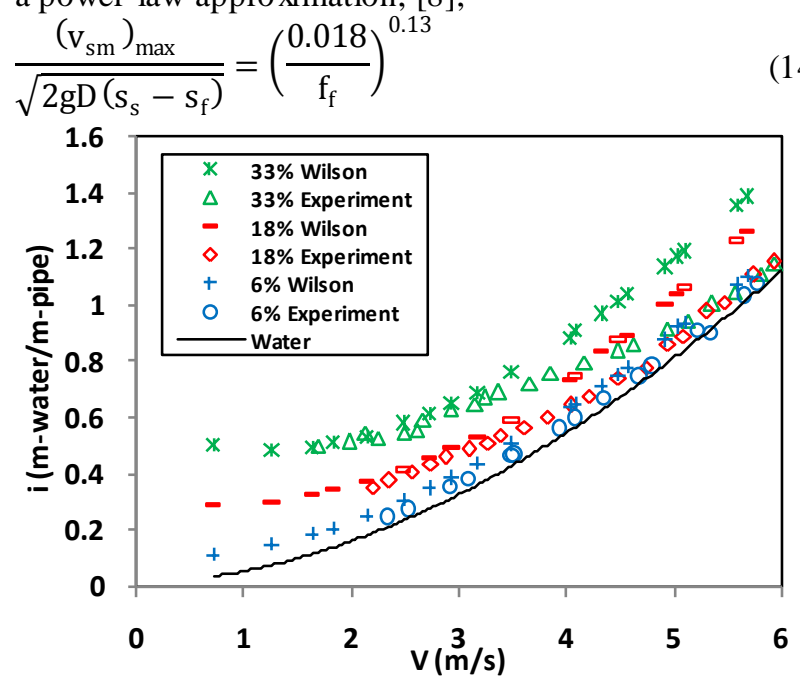

Figure (14) Wilson and experimental results of mediu m sand slurry at different concentrations



Figure (15) Wilson and experimental results of fine sand slurry at different concentrations

Figures (13)-(15) present the experimental and Wilson resistance curves of coarse, mediu $m$ and fine sand slurries at different concentrations. The general trends is that increasing the mean slurry velocity leads to increasing the hydraulic gradient at the same solid concentration and at the same slurry mean velocity the hydraulic gradient increases as the solid concentration increases. The solid effect decreases as the slurry velocity increases. The experimental and numerical Wils on hydraulic gradients for coarse sand slurry are typically matching or all solid concentrations and at all velocity range.

At high slurry velocity there are very small and insignificant deviations between the two gradients to be neglected (trivial over prediction of Wilson results). Wilson is the perfect model to describe the coarse particle slurry behaviour. From literature Wilson model is appropriate for stratified slurries so the studied coarse particle slurry is stratified regime.

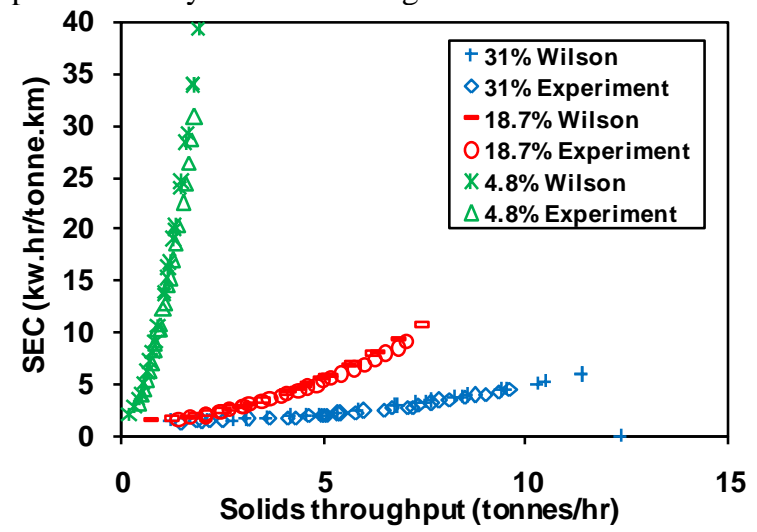

Figure (16) Wilson and experimental specific energy consumptions of coarse slurry at different concentrations

Experimental hydraulic gradient and numerical one of the medium sand slurry coincide at low slurry velocity and have a certain difference at high velocity $(\mathrm{V}>3.5 \mathrm{~m} / \mathrm{s}$ ) at high solid concentration slurries $\left(\mathrm{C}_{\mathrm{v}}=33 \%\right.$ and $\left.18 \%\right)$ but at low concentration slurries $\left(\mathrm{C}_{\mathrm{v}}=6 \%\right)$ there are relative analogy in behaviours with water. Wilson succeeded to simulate the medium sand behaviour at certain circumstances. The slope of the experimental curves decreases as the slurry velocity increases but the slope of the numerical curves increases.

There is a remarkable difference between the experimental and numerical hydraulic gradients for fine particle size slurries especially at high concentrations but at low solid concentration there is a comparative analogy. Wilson is not suitable correlation for fine sand slurries investigation due to this fine sand slurry is dreamy to has stratified regime.

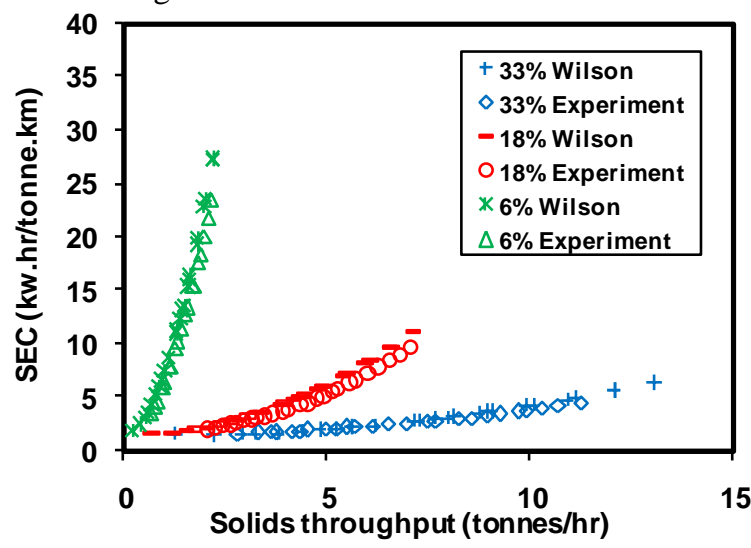

Figure (17) Wilson and experimental energy consumptions of mediu m slurry at diffe rent concentrations

Figures (16)-(18) show experimental results and Wilson relation of the specific energy consumption with solids throughput for coarse, medium, and fine sand slurries at different solid concentrations. Figures illus trate that at any 
concentration the experimental and Wilson energy consumptions increases as solids throughput increases.

There is high agreement between the experimental and numerical calcu lations of specific energy consumptions of coarse sand slurries at all solid concentration. The agreement decreases for medium and fine slurries especially at high solid concentrations. As the solids concentration increase the specific energy consumption, for the same solids throughput, decreases for all sand slurries. Decreasing of specific energy consumption means the increasing of the pipeline hydro-transport efficiency of the sand slurries.

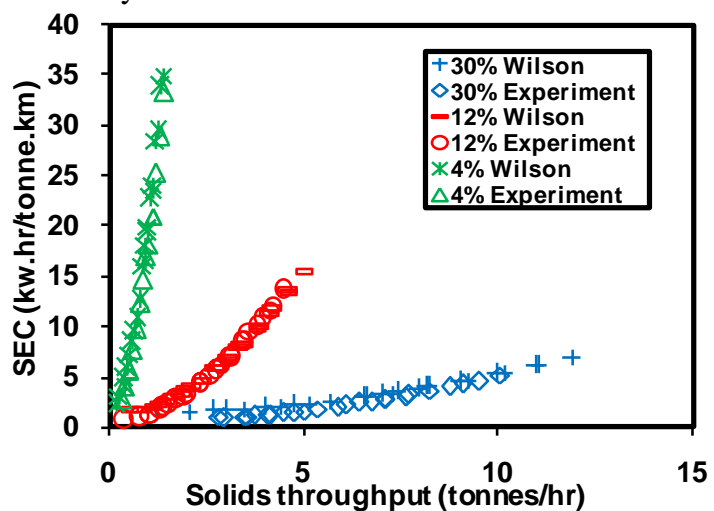

Figure (18) Wilson and experimental specific energy consumptions of fine slurry at different concentrations

Wilson behaviors depending on the variation of the solid particle size could be shown obviously in figures (19)-(20) by comparing the behaviours of coarse, medium and fine sand slurries at nearly the same solid concentration. Investigating of behavior shows that there is no effect of solid particle size.



Figure (19) Wilson resistance curves of different particle



Figure (20) Wilson resistance curves of different particle sizes slurries

\subsection{Wilson-Addie-Selgren-Clift (WASC) Correlation}

In heterogeneous slurry, only a portion of the solids is carried out by granular contact (contact load), another portion is suspended by a fluid, (suspended load). The presence of suspended load reduces the ratio of $\frac{i_{m}-i_{f}}{S_{m}-S_{f}}$ to value less than for the fully-stratified flow. The centralvalue parameter will be denoted by $V_{50}$, signifying the value of mean velocity $\mathrm{V}_{\mathrm{m}}$ at which the stratification ratio is $0.50,[26]$.

$\mathrm{V}_{50}=\mathrm{w}_{50}\left(\frac{2}{\mathrm{f}_{\mathrm{f}}}\right)^{\frac{1}{2}} \cosh \left(\frac{60 \mathrm{~d}_{50}}{\mathrm{D}}\right)$

As noted above, the particle-associated velocity, w, can be considered as.

$\mathrm{w}=0.9 \mathrm{v}_{\mathrm{t}}+2.7\left(\frac{\left(\rho_{\mathrm{s}}-\rho_{\mathrm{f}}\right) g \mu_{\mathrm{f}}}{\rho_{\mathrm{f}}^{2}}\right)^{\frac{1}{3}}$
$\frac{\mathrm{i}_{\mathrm{m}}-\mathrm{i}_{\mathrm{f}}}{\mathrm{S}_{\mathrm{m}}-\mathrm{S}_{\mathrm{f}}}=0.22\left(\frac{\mathrm{V}_{50}}{\mathrm{~V}_{\mathrm{m}}}\right)^{\mathrm{M}}$

It has been observed that the exponent $\mathrm{M}$ is often near 1.7 for narrow particle grading, and decreases as the grading becomes broader.

$M=\left(0.25-13 \sigma_{\mathrm{s}}^{2}\right)^{-\frac{1}{2}}$
$\sigma_{\mathrm{s}}=\log _{10}\left(\frac{\mathrm{w}_{85} \cosh \left(\frac{60 \mathrm{~d}_{85}}{\mathrm{D}}\right)}{\mathrm{w}_{50} \cosh \left(\frac{60 \mathrm{~d}_{50}}{\mathrm{D}}\right)}\right)$

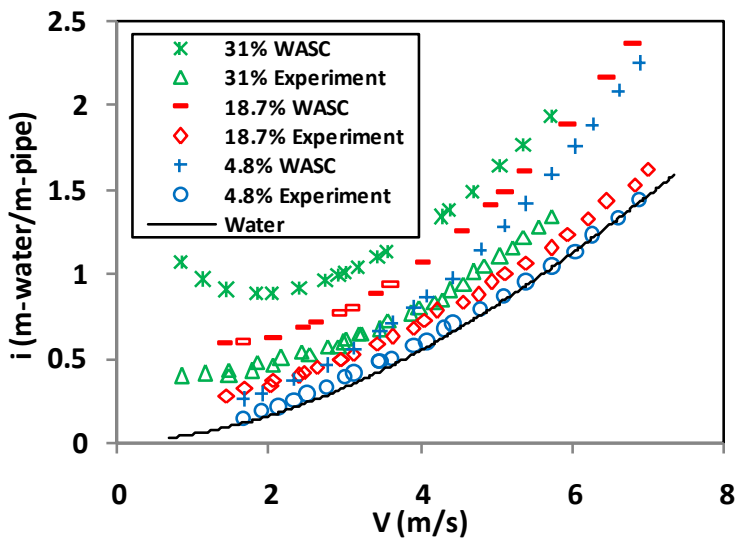

Figure (21) WASC and experimental results of coarse sand slurry at different concentrations

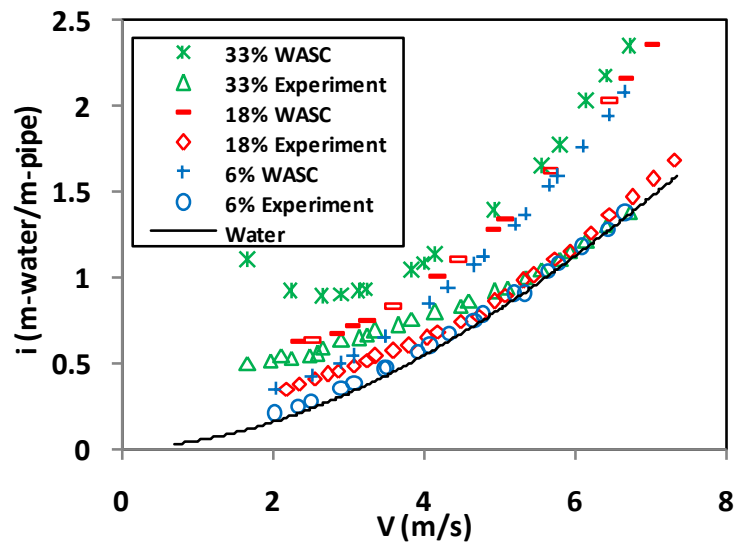

Figure (22) WASC and experimental results of med iu $\mathrm{m}$ sand slurry at different concentrations 
It must be remembered the proviso that a calculated $\mathrm{M}$ larger than 1.7 must be reduced to that value so that the equation will be modified to;

$$
\mathrm{M}=\min \left[\left(0.25-13 \sigma_{\mathrm{s}}^{2}\right)^{-\frac{1}{2}}, 1.7\right]
$$

Figures (21)-(23) present the experimental and the WASC resistance curves of coarse, medium and fine sand slurries at different solid concentrations. Increasing the slurry velocity at certain solid concentration leads to increasing of the pressure losses. From figures there is a large difference between experimental and computational behaviours at all concentrations and particle sizes so this model can't clarify the studied cases. The figures show at the same slurry mean velocity the hydraulic gradient increases as the solid concentration of the slurry increases. The smallest the solid concentration of the WASC resistance curves is the highest curve slope. There are great differences between WASC resistance curves at low slurry velocity but they marry up at high slurry velocity.



Figure (23) WASC and experimental results of fine sand slurry at different concentrations

\subsection{Turian and Yuan Correlation}

The flow takes the form of different regimes. Four distinct regimes were found (flow with stationary bed, flow with moving bed, heterogeneous suspension and homogeneous suspension) in slurry flow depending upon several flow parameters.

As regime identification is an important criterion for slurry pipeline design to select suitable correlation of pressure drop for that regime, a method has been proposed to identify the regime using Turian and Yuan's approach. The pressure drop correlations are applicable to a particular regime for which they were developed. The correlations show ill prediction of pressure drop when they apply for other regimes. Thus regime identification becomes important for slurry pipeline design as they are the prerequisite to select suitable pressure drop correlation in that regime. The main problem arises because of difficulty to define the boundaries between the flow regimes. These boundaries are poorly defined because these are based on visual observations of particle motions. The approach of Turian and Yuan claims to provide a completely self consistent definition of the flow regime boundaries. Turian and Yuan established that the excess pressure gradient in each flow regime can be correlated using an equation of the form:
$\mathrm{f}_{\mathrm{sl}}-\mathrm{f}_{\mathrm{f}}=\mathrm{K}^{\prime} \mathrm{C}^{\alpha} \mathrm{f}_{\mathrm{f}}{ }^{\prime \prime} \mathrm{C}_{\mathrm{D}}^{*}{ }^{\mathrm{\gamma}} \mathrm{F}_{\mathrm{r}}^{\delta}$

The coefficients $\mathrm{K}^{\prime}, \alpha^{\prime}, \alpha^{\prime \prime}, Y$ and $\delta$ have values that are specific to each flow regime. The best available values of these coefficients in each flow regime are given by, [26];

$$
\begin{aligned}
& \mathrm{C}_{\mathrm{D}}^{*}=\frac{432}{\Phi_{1}^{*}}\left(1+0.047\left(\Phi_{1}^{*}\right)^{\frac{2}{3}}\right)+\frac{0.517}{1+154\left(\Phi_{1}^{*}\right)^{-\frac{1}{3}}} \\
& \Phi_{1}^{*}=\left(\mathrm{d}_{\mathrm{p}}^{*}\right)^{3}=\frac{4}{3} \frac{\left(\rho_{\mathrm{s}}-\rho_{\mathrm{f}}\right) \rho_{\mathrm{f}} \mathrm{d}^{3} \mathrm{~g}}{\operatorname{le}_{\mathrm{f}}^{2}} \\
& \mathrm{~F}_{\mathrm{r}}=\frac{\mathrm{V}_{\mathrm{m}}^{2}}{\mathrm{gD}(\mathrm{S}-1)}
\end{aligned}
$$



Figure (24) Turian resistance curves for different regimes of coarse sand at solid concentration $31 \%$

\section{Sliding bed (regime 0)}

$\mathrm{f}_{\mathrm{sl}}-\mathrm{f}_{\mathrm{f}}=12.13 \mathrm{C}^{0.7389} \mathrm{f}_{\mathrm{f}}^{0.7717} \mathrm{C}_{\mathrm{D}}^{*-0.4054} \mathrm{~F}_{\mathrm{r}}{ }^{-1.096}$

\section{Moving bed (regime 1)}

$\mathrm{f}_{\mathrm{sl}}-\mathrm{f}_{\mathrm{f}}=107.1 \mathrm{C}^{1.018} \mathrm{f}_{\mathrm{f}}{ }^{1.046} \mathrm{C}_{\mathrm{D}}^{*-0.4213} \mathrm{~F}_{\mathrm{r}}^{-1.354}$

\section{Heterogeneous suspension (regime 2)}

$\mathrm{f}_{\mathrm{sl}}-\mathrm{f}_{\mathrm{f}}=30.11 \mathrm{C}^{0.868} \mathrm{f}_{\mathrm{f}}^{1.200} \mathrm{C}_{\mathrm{D}}^{*}{ }^{-0.1677} \mathrm{~F}_{\mathrm{r}}{ }^{-0.6938}$

\section{Homogeneous suspension (regime 3)}

$\mathrm{f}_{\mathrm{sl}}-\mathrm{f}_{\mathrm{f}}=8.538 \mathrm{C}^{0.5024} \mathrm{f}_{\mathrm{f}}^{1.428} \mathrm{C}_{\mathrm{D}}^{* 0.1516} \mathrm{~F}_{\mathrm{r}}^{-0.3531}$

$\Delta \mathrm{P}_{\mathrm{f}, \mathrm{sl}}=2 \mathrm{f}_{\mathrm{sl}} \rho_{\mathrm{w}} \mathrm{V}_{\mathrm{m}}^{2} \frac{\mathrm{L}}{\mathrm{D}}$

$\mathrm{i}_{\mathrm{m}}=\frac{\Delta \mathrm{P}_{\mathrm{f}, \mathrm{sl}}}{\operatorname{Lg} \rho_{\mathrm{w}}}=2 \mathrm{f}_{\mathrm{sl}}(\mathrm{S}-1) \mathrm{F}_{\mathrm{r}}$

Some authors prefer to use $\rho_{\mathrm{sl}}$ rather than $\rho_{\mathrm{w}}$.

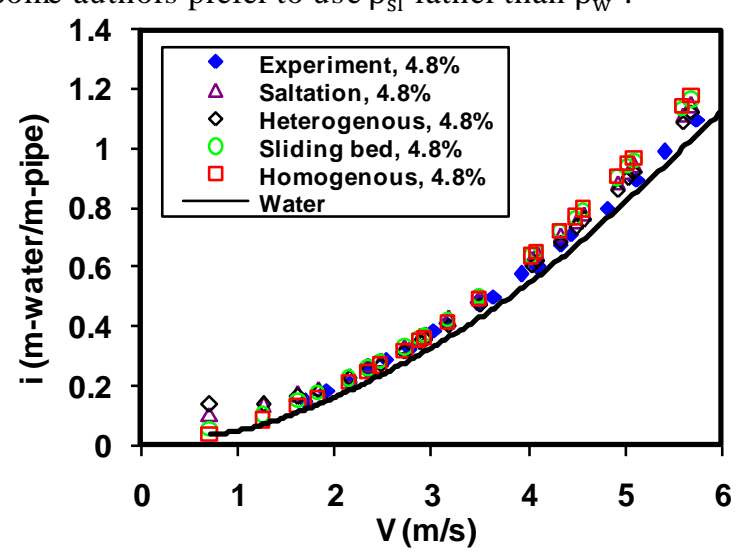

Figure (25) Turian resistance curves for different regimes of coarse sand at solid concentration $4.8 \%$ 
Figure (24) shows all regimes that can be obtained from Turian model of the flow of coarse slurry at solid concentration $\left(\mathrm{C}_{\mathrm{v}}=31 \%\right)$ with experimental results. The figure illus trates that at low slurry velocity $(\mathrm{v}<1.5 \mathrm{~m} / \mathrm{s})$ the flow regime is fully stratified (sliding bed), as the slurry velocity increases $(1.5 \mathrm{~m} / \mathrm{s}<\mathrm{v}<3.5 \mathrm{~m} / \mathrm{s})$ the regime become saltation, finally as the slurry velocity increases $(3.5 \mathrm{~m} / \mathrm{s}<\mathrm{v})$ the regime beco me heterogeneous. The results obtained from this figure obey the theoretical results. At low slurry velocity the high concentrated coarse sand particles settled in the bottom of the pipe forming the sliding bed regime, as the slurry velocity increase the particles of the solid will be transported in saltation to heterogeneous regimes.

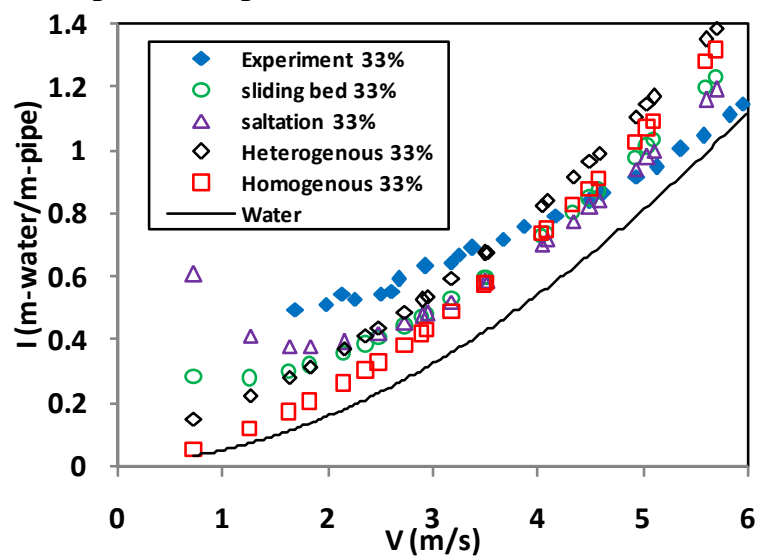

Figure (26) Turian resistance curves for different regimes of medium sand at solid concentration $33 \%$

Figure (25) shows all regimes that can be obtained from Turian model in the flow of coarse slurry at solid concentration $\left(\mathrm{C}_{\mathrm{v}}=4.8 \%\right)$ with experimental results. In this figure, all trends are coinciding such that it is difficult to identify the specified flow regime at certain velocity. With deeply focusing it's found that at $\mathrm{v}<3 \mathrm{~m} / \mathrm{s}$ the regime is stratified with increasing the velocity $v>3 \mathrm{~m} / \mathrm{s}$ the regime become heterogeneous. Comparing this figure with the previous one explains that increasing concentration enhance the interference between the solid particles.



Figure (27) Turian resistance curves for different regimes of medium sand at solid concentration $12 \%$

Figure (26) shows all regimes that can be obtained from Turian model calculations in the flow of medium sand particle slurry at solid concentration $\left(\mathrm{C}_{\mathrm{v}}=33 \%\right)$ with experimental results. In this figure, at the majority of velocity range the closest regime to the experimental one is the heterogeneous regime but at very low velocity, $\mathrm{v}<2.5 \mathrm{~m} / \mathrm{s}$, the saltation is most possible regime and at high slurry velocity the experimental trend of the medium sand slurry at solid concentration $33 \%$ unusual such that it cant be explained by any Turian corre lations.

Figure (27) shows all regimes that can be obtained from Turian model calculations in the flow of medium sand particle slurry at solid concentration $\left(\mathrm{C}_{\mathrm{v}}=12 \%\right)$ with experimental results. In this figure, approximately at the whole velocity range it's clear that the closest regime to the experimental one is the heterogeneous regime but at very low velocity, $\mathrm{v}<2.2 \mathrm{~m} / \mathrm{s}$, the stratification is most possible regime.



Figure (28) Turian resistance curves for different regimes of fine sand at solid concentration $30 \%$

Figure (28) shows all regimes that can be obtained from Turian model in the flow of fine sand particle slurry at the solid concentration $\left(\mathrm{C}_{\mathrm{v}}=30 \%\right)$. In this figure, at low slurry velocity range $\mathrm{v}<1.8 \mathrm{~m} / \mathrm{s}$ there are irregular random variations of regimes. These unsystematic variations remain to small velocity range. For slurry velocity $\mathrm{v}>1.8 \mathrm{~m} / \mathrm{s}$ the heterogeneous regime is the closest regime to the experimental one. Usually, all practical hydrotransport are carried out in heterogeneous suspension regime because of lowest pressure drop and subsequent less power require ment.

\section{COMPARISON BETWEEN CORRELATIONS OF HYDRAULIC GRADIENT}



Figure (29) Resistance curves of all models for coarse sand slurry at solid concentration $31 \%$ 
Figure (29) illustrates the resistance curves of the coarse sand slurry at high solid concentration $\left(\mathrm{C}_{\mathrm{v}}=31 \%\right)$. All resistance curves of different models have the general trend in which the head loss increases as the slurry velocity increases. The highest solid effect of the different resistance curves belongs to the WASC model. The correlation that matches with the experimental results is the Wilson and Addie model at all velocity range. As a result, it's recommended to choose Wilson and Addie model to simulate or to design the flow of coarse sand particle slurry $\left(\mathrm{d}_{50}=1.4 \mathrm{~mm}\right)$ with solid concentration $31 \%$ in pipe dia meter $26.8 \mathrm{~mm}$.



Figure (30) Resistance curves of all models for coarse sand slurry at solid concentration $4.8 \%$

Figure (30) shows the resistance curves of the coarse sand particle at low solid concentration $\left(\mathrm{C}_{\mathrm{v}}=4.8 \%\right)$. At this extremely low solid concentration the most behaviors are coincide and nearly approach to the water behavior so the distinction between these trends is very difficult in this overlap trends. The correlation that matches with the experimental results is Wilson and Durand models. It can be concluded that Wilson correlation of head loss calculation must be used to optimize the design of pipeline (has internal pipe diameter $26.8 \mathrm{~mm}$ ) has a coarse slurry flow $\left(\mathrm{d}_{50}=1.4 \mathrm{~mm}\right)$ at any solid concentration (from $\mathrm{C}_{\mathrm{v}}=4.8 \%$ to $31 \%$ ) to obtain maximum efficiency and minimum power consumptions. At low solids concentration of this coarse sand slurry Durand correlation might be used in the calculations. This case of coarse slurry flow has a stratified regime at low velocity and heterogeneous regime at high slurry velocity (these velocities differ accord ing to solid concentration).

Figure (31) illustrates the resistance curves of the med iu m sand slurry at high solid concentration $\left(\mathrm{C}_{\mathrm{v}}=33 \%\right)$. The experimental solid effect decreases as the slurry velocity increases. The correlation for head loss calculation that matches with the experimental results especially at low slurry velocity is the Wilson model $(\mathrm{v}<3.5 \mathrm{~m} / \mathrm{s})$. At high velocity range $(\mathrm{v}>4.5 \mathrm{~m} / \mathrm{s})$ the $\mathrm{W}$ ils on model becomes not suitable and the matching become between the experimental and Durand model.

For the slurry velocity range, $3.5 \mathrm{~m} / \mathrm{s}<\mathrm{v}<4.5 \mathrm{~m} / \mathrm{s}$, there is no matching between the experimental results and any of correlations so the most approximated correlation for this velocity range either Wilson or Durand. If it's desired to simulate the medium sand particle slurry $\left(\mathrm{d}_{50}=0.7 \mathrm{~mm}\right)$ with solid concentration $33 \%$ in pipeline diameter
$26.8 \mathrm{~mm}$ it's recommended to choose Wilson model at low slurry velocity and choose Durand at high slurry velocity.

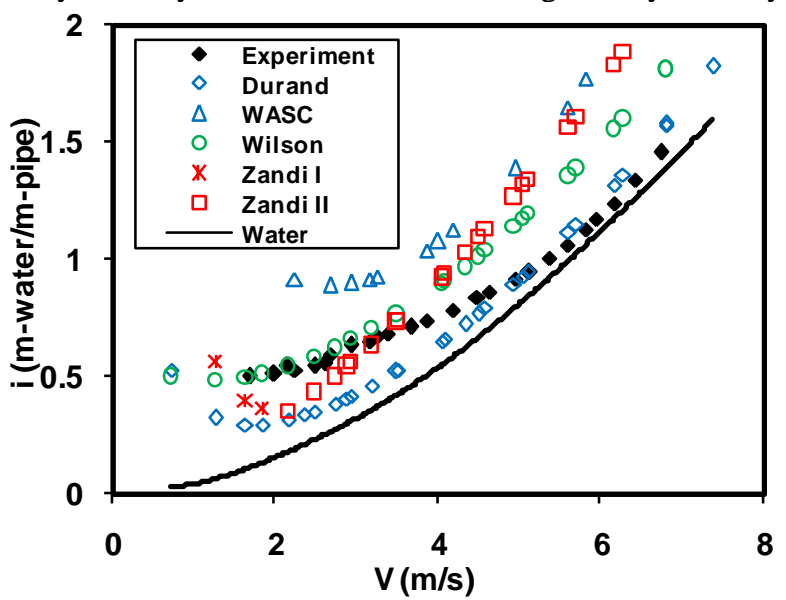

Figure (31) Resistance curves of all models for medium sand slurry at solid concentration $33 \%$

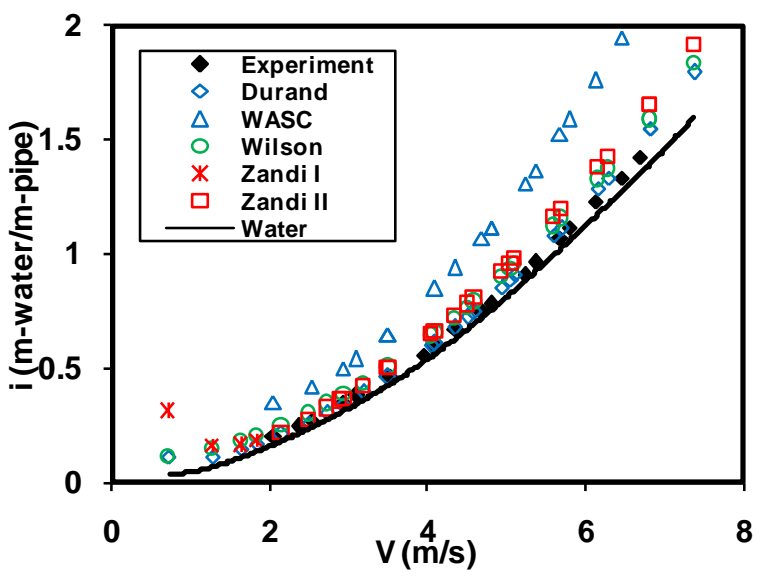

Figure (32) Resistance curves of all models for medium sand slurry at solid concentration $6 \%$

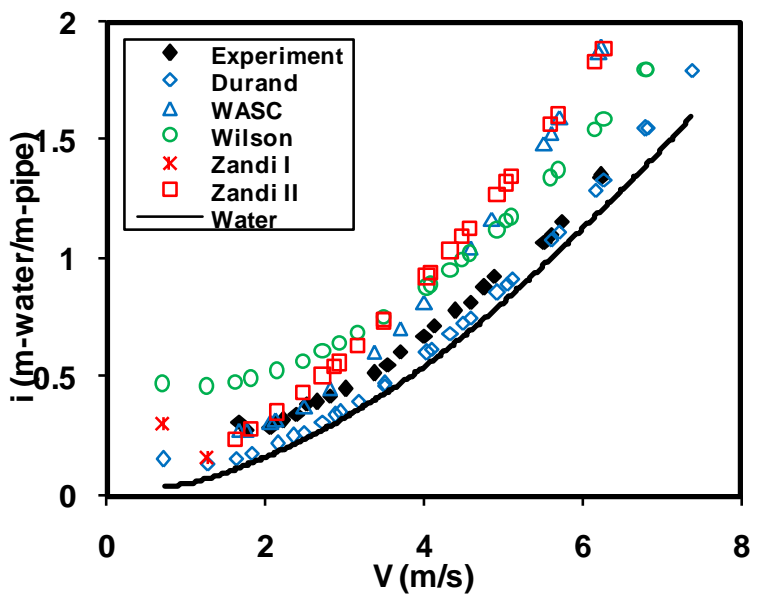

Figure (33) Resistance curves of all models for fine sand slurry at solid concentration $30 \%$

Figure (32) illustrates the resistance curves of the medium sand particle at low solid concentration $\left(\mathrm{C}_{\mathrm{v}}=6 \%\right)$. Most behaviors of different correlations are coincide and highly approached to the water behaviour. Also at this low solid concentration the experimental results are coincide with water behavior. The correlation that matches with the experimental results is the Durand and Wilson models respectively. So that in the simulation of 
the medium sand slurry $\left(\mathrm{d}_{50}=0.7 \mathrm{~mm}\right)$ that flows in pipeline diameter $26.8 \mathrm{~mm}$ and according to the solid concentration (from $\mathrm{C}_{\mathrm{v}}=6 \%$ to $33 \%$ ) either Wilson or Durand or both of them should be used.

Figure (33) illustrates the resistance curves of the fine slurry at high solid concentration $\left(\mathrm{C}_{\mathrm{v}}=30 \%\right)$. The correlation that matches with the experimental results is Durand model. Durand is the preferable correlation to fine sand slurry flow heterogeneously in pipeline diameter $26.8 \mathrm{~mm}$ at different solid concentrations from $\mathrm{C}_{\mathrm{v}}=4 \%$ to $30 \%$.

\section{CONCLUSIONS}

The aim of this study is to establish a relationship between some pressure gradient equations for slurry flow and the experimental data obtained for the flow of three patches of sand particles $(0.2,0.7$ and $1.4 \mathrm{~mm})$ in water carrier fluid at different concentrations (4\%-33\%) and velocities (up to $7 \mathrm{~m} / \mathrm{s}$ ). The variables are determined in accordance with the experimental conditions. The solid particles that are present in the slurry generate additional momentum transfer that leads to increase in the pressure drop.

The sand slurries have different trends in the development of the hydraulic gradient dependence on the mean velocity but customarily the hydraulic gradient increases as the mean velocity increases. At the same slurry mean velocity, as the solids concentration increases the flow friction loss generally increases, and the specific energy consumption for the same solids throughput decreases. With the increasing of the particle size the friction loss and specific energy consumption mostly increase.

Wilson and Durand correlations are the most appropriate models to simulate the experimental results for the three patches of sand slurries at different concentrations and velocities. Wilson is applicable for coarse sand slurry at different concentrations. For medium slurry at high concentration Wilson is valid for low velocity $(\mathrm{v}<3.5 \mathrm{~m} / \mathrm{s})$ and Durand is lawful for high velocity $(\mathrm{v}>4.5 \mathrm{~m} / \mathrm{s})$ but at low concentration either Wilson or Durand is official at all velocity range. Durand is authorized for fine slurry at all concentrations. The matching between the experimental with Durand and Wilson specific energy consumptions emphasize the adaptation of these models to simulate the studied system.

Usually, all practical hydro-transport of the studied systems are carried out in heterogeneous regime at the majority of velocity range because of lowest power requirement but at very low slurry velocity the stratified regime is the dominant one.

It is observed that not all expressions developed for the slurry flow simulate the experimental results. Since the expressions developed by various authors for slurry pressure gradients are based on the empirical correlation of the data collected from experiments conducted in a laboratory, they can be expected to be valid only for conditions similar to those of the conducted experiments, and their extrapolations outside mentioned areas might yield incorrect results. Therefore, one should be careful in using empirical formulas and special tests should be conducted to determine accurate flow regimes and the relationships between the bulk velocity and pressure gradients for each specific situation.

\section{LIST OF SYMBOLS}

C Delivered concentration of the solid [-]

$C_{D} \quad$ Drag coefficient [-]

$\mathrm{C}_{\mathrm{D}}^{*} \quad$ Particle drag coefficient at terminal velocity [-]

$\mathrm{C}_{\mathrm{v}} \quad$ Volumetric concentration of solid [-]

$\mathrm{C}_{\mathrm{vd}} \quad$ Delivered volu metric concentration [-]

$\mathrm{d} \quad$ Partic le diameter [m]

$\mathrm{d}_{50} \quad$ Mass median particle diameter [m]

$\mathrm{d}_{85}$ Diameter of which $85 \%$ of particles are finer [m]

D Pipe internal diameter $[\mathrm{m}]$

f Darcy friction factor of the fluid [-]

$\mathrm{f}_{\mathrm{f}} \quad$ Fanning friction factor of the carrier fluid [-]

$\mathrm{f}_{\mathrm{sl}} \quad$ Fanning friction factor of the slurry [-]

$\mathrm{F}_{\mathrm{r}} \quad$ Froude number [-]

$i_{f} \quad$ Hydraulic gradient of the fluid [-]

$\mathrm{i}_{\mathrm{m}} \quad$ Hydraulic gradient of the mixture flow [-]

$\mathrm{K}$ Durand and Zandi empirical coefficient [-]

$\mathrm{K}^{\prime}$ Coefficient of Turian and Yuan correlation [-]

M Particle distribution parameter [-]

n Durand and Zandi e mpirical coefficient [-]

$\mathrm{N}_{\mathrm{e}}$ Zandi and Govatos index number [-]

$\mathrm{Re}_{\mathrm{N}} \quad$ Newtonian fluid Reynolds's number [-]

$\mathrm{S}_{\mathrm{m}} \quad$ Relative density of the delivered mixture [-]

$\mathrm{S}_{\mathrm{S}} \quad$ Relative density of the solid particle [-]

SEC Specific Energy Consumption [kw.hr/tonne.km]

$\mathrm{V}$ Velocity $[\mathrm{m} / \mathrm{s}]$

$\mathrm{V}_{\mathrm{m}} \quad$ Mean velocity $[\mathrm{m} / \mathrm{s}]$

$\mathrm{V}_{\mathrm{sm}}$ Maximu $\mathrm{m}$ value of limit deposition velocity $[\mathrm{m} / \mathrm{s}$ ]

$\left(\mathrm{V}_{\mathrm{sm}}\right)_{\max }$ Value of $\mathrm{V}_{\mathrm{sm}}$ for fully-stratified flow with shear layer $[\mathrm{m} / \mathrm{s}]$

$\mathrm{v}_{\mathrm{t}}$ Terminal settling velocity of the solid particle $[\mathrm{m} / \mathrm{s}]$

$\mathrm{V}_{50} \quad$ Velocity at midpoint from fully stratified and heterogeneous $[\mathrm{m} / \mathrm{s}]$

$w_{50}$ Terminal settling velocity of particle size $d_{50}[\mathrm{~m} / \mathrm{s}]$

$\mathrm{w}_{85}$ Terminal settling velocity of particle size $d_{85}[\mathrm{~m} / \mathrm{s}]$

\section{Greek Letters}

$\varepsilon \quad$ Specific roughness $[\mathrm{m} / \mathrm{s}]$

$\mu_{\mathrm{f}} \quad$ Flu id dynamic viscosity $[\mathrm{kg} / \mathrm{m} . \mathrm{s}]$

$\rho_{\mathrm{f}} \quad$ Flu id density $\left[\mathrm{kg} / \mathrm{m}^{3}\right]$

$\rho_{\mathrm{s}} \quad$ Particle density $\left[\mathrm{kg} / \mathrm{m}^{3}\right]$

$\Phi \quad$ Excess pressure gradient [-]

$\Phi_{1}^{*} \quad$ Dimensionless number, equation (23) [-]

$\Psi$ Dimensionless number, equations $(7,8,11)[-]$

$\alpha$ Coefficient of Turian corre lation, equation (21) [-]

$\alpha^{\prime \prime} \quad$ Coefficient of Turian corre lation, equation (21) [-]

$\mathrm{y}$ Coefficient of Turian corre lation, equation (21) [-]

$\delta$ Coefficient of Turian corre lation, equation (21) [-]

$\sigma_{\mathrm{s}} \quad$ Normal stress of solid (intergranular) $\left[\mathrm{kg} / \mathrm{m} . \mathrm{s}^{2}\right]$

\section{REFERENCES}

[1] Durand, R. and Condolios, E., "Experimental Study of the Hydraulic Transport of Coal and Solids Materials in Pipes", Proc. Colloq. on the hydraulic transport of coal, National Coal Board (UK), paper 
IV, 1952, 39-55.

[2] Durand, R., "Basic Relationships of the Transportation of Solids in Pipes-Experimental Research", proc. $5^{\text {th }}$ Minneapolis Int. Hydraulics Convent., Minneapolis, MN, 1953, 89-103.

[3] Zandi, I. and Govatos, G., "Heterogeneous Flow of Solids in Pipelines", Proc. Amer. Soc. Civil Eng., 93. No. HY7, 1967, 145-159.

[4] Zandi, I., "Advances in Solid Liquid Flow in Pipes and its Application", Pergamon Press, N.Y, 1971.

[5] Turian, R.M. and Yuan, T.F., "Flow of Slurries in Pipelines", AICh.E J. 23, 1977, 232-243.

[6] Wilson, K.C., "Analysis of Contact-Load Distribution and Application to Deposition Limit in Horizontal Pipes", J. Pipe lines 4, 1984, 171-176.

[7] Turian, R.M, Hsu, F.L. and Ma, T.W., "Estimation of the Critical Velocity in Pipeline Flow of Slurries", Powder Technology 51, 1987, 35-47.

[8] Wilson, K.C., Addie, G.R., Sellgren, A. and Clift, R., "Slurry Transport Using Centrifugal Pumps", Elsevier Applied Science, 1992, London.

[9] Sundqvist, A., Sellgren, A., and Addie, G., "Slurry Pipeline Friction Losses for Coarse and High Density Industrial Products", Powder Technology 89, 1996, 19-28.

[10] Sundqvist, A., Sellgren, A. and Addie, G., "Pipeline Friction Losses of Coarse Sand Slurries", Powder Technology 89, 1996, 9-18.

[11] Liu, J., and Galvin, K.P., "Mechanics of a Concentrated Slurry Feed System", Powder Technology 102, 1999, 227-234.

[12] El-Nahhas, K., "Hydraulic Transport of Dense Fine-Grained Suspensions", Ph.D. thesis, Faculty of Engineering at Port Said, Port Said University, 2002.

[13] Wilson, K.C., Addie, G.R, Clift, R. and Sellgren, A., "Operating Points for Pipelines Carrying Concentrated Heterogeneous Slurries", Powder Technology 123, 2002, 19-24.

[14] Kaushal, D.R. and Tomita, Y., "Solids Concentration Profiles and Pressure Drop in Pipeline Flow of Multisized Particulate Slurries", International Journal of Multiphase Flow 28, 2002, 1697-1717.

[15] Miedema, S.A. and Matousek, V., "Reconstruction of and Numerical Sensitivity Analysis on Wilson Model for Hydraulic Transport of Solids in Pipelines", WEDA Journal of Dredging Engineering, March 2002.

[16] Kausha, D.R. and Yuji Tomita, "Comparative Study of Pressure drop in Multisized Particulate Slurry Flow Through Pipe and Rectangular Duct", International Journal of Multiphase Flow 29, 2003, 1473-1487.

[17] Matousek, V., "Research Developments in Pipeline Transport of Settling Slurries", Powder Technology $156,2005,43-51$.

[18] Matousek, V., "Research Developments in Pipeline Transport of Settling Slurries", Powder Technology $156,2005,43-51$.

[19] Kaushal, D.R., Sato, K., Toyota, T. and Tomita, Y., "Effect of Particle Size Distribution on Pressure Drop and Concentration Profile in Pipeline flow of
Highly Concentrated Slurry", International Journal of Multiphase Flow 31, 2005, 809-823.

[20] Matousek, V.," Interaction of Slurry Pipe Flow with a Stationary Bed", $17^{\text {th }}$ International Conference on the Hydraulic Transport of Solids, 2007.

[21] Deniz Ulusarslan, "Comparison of Experimental Pressure Gradient and Experimental Relationships for the Low Density Spherical Capsule Train with Slurry Flow Relationships", Powder Technology 185, 2008, 170-175.

[22] El-Nahhas, K., Gad El-Hak, N., Abou Rayan, M. and El-Sawaf, I., "Effect of Particle Size Distribution on the Hydraulic Transport of Settling Slurries", Thirteenth International Water Technology Conference, 2009, 463-474, Egypt.

[23] Lahiri, S.K. and Ghanta, K.C., "Study on Slurry Flow Modeling in Pipeline", Ph.D thesis, National Institute of Technology, Durgapur, India, 2009.

[24] Jie Wua, Graham, L. and Parthasarathy, R., "Energy Efficient Slurry Holding and Transport", Minerals Engineering 23, 2010, 705-712.

[25] Venkatesan, M., Sarit K. Das and Balakrishnan, A.R., "Effect of Diameter on Two-Phase Pressure Drop in Narrow Tubes", Experimental Thermal and Flu id Science 35, 2011, 531-541.

[26] R. P. King, "Introduction to Practical Fluid Flow", University of Utah, 2002, ISBN 0750648856. 